\title{
Drug Review on Siddha Drug - Thirikadugadhi Mandooram
}

\author{
Shakthi Priya $\mathbf{M}^{1}$, Essakky Pandian $G^{2}$ \\ ${ }^{1}$ PG Scholar, Department of Gunapadam, ${ }^{2}$ Reader, Department of Gunapadam, \\ Government Siddha Medical College, Palayamkottai, Tirunelveli, Tamil Nadu, India. \\ Corresponding Author: Shakthi Priya M
}

\begin{abstract}
Siddha system is an oldest system of traditional medicine originating in ancient Tamilnadu in South India. In Siddha system, medicine includes the herbal products, inorganic substances and animal products that lead to different formulations ranging from low shelf life drug to high shelf life drug. Thirikadugadhi Mandooram is a poly herbo-mineral formulation used to treat Anaemia, Jaundice, Anasarca, Fever, Tuberculosis. This review describes the phytochemicals, pharmacological action and medicinal uses of the part of each ingredient used in this formulation. Ingredients of the formulation and their pharmacological action in various research studies are discussed in this review. The details were collected from different Siddha texts and electronic databases. In conclusion the results of the review revealed that the pharmacological action and the medicinal uses of drug were perfectly matched with each ingredients of formulation.
\end{abstract}

Keywords: Siddha system, Thirikadugadhi Mandooram, review

\section{INTRODUCTION}

Siddha medicine is an oldest system of traditional medicine originating in ancient Tamilnadu in south India. Palm leaf manuscripts say that the Siddha system was $1^{\text {st }}$ described by Lord Shiva to his wife Parvati. The concept of siddha medicine is based on 96 thathuvas. According to the siddha medicine system, diet and lifestyle plays a major role in health and in curing disease. This system believes that all objects in the universe including human body are composed of basic elements namely earth, water, fire, air and space.

In siddha medicine, there are 32 internal and 32 external medicinal preparations are available for the treatment. Mathirai (pills) is the one form of internal medicine in which powdered raw drugs are triturated with the juice of leaves or decoction, ghee or honey or types of milk. They are rolled into different size of pills dried and stored.
The drug review of Thirikadugadhi Mandooram is a poly herbo-mineral formulation gives evidence for its therapeutic actions mentioned in literatures. It has 15 ingredients among all only 2 metal drugs and other all from plant origin and detoxification process of all ingredients should be done before the preparation of final medicine. This review describes the phytochemicals, pharmacological action and medicinal uses of the part of each ingredient used in this formulation.

\section{MATERIALS AND METHODS}

Research design: Drug review on literature

Research type: Literature review

Research period: 3 months

Literature collected from:

Thiyagarajan.K, GunapadamThadhu-Jeeva vaguppu (part - 2\&3) published by Department of Indian medicine \& Homeopathy, Chennai -106, pg no:200. 


\section{INGREDIENTS OF DRUG:}

Chukku (Zingiber officinale.Linn.)

Milagu (Piper nigrum.Linn.)

Thippili (Piper longum.Linn.)

Kadukkai (Terminalia chebula.Linn.)

Nellivattral (Phyllanthus emblica.Linn.)

Thandrikai (Terminalia bellerica.Linn.)

Elam (Elettaria cardamomum.Linn.)

Saadhikai (Myristica fragrans.Linn.)

Krambu (Syzygium aromaticum.Linn.)

Kattaathipoo (Bauhinia tomentosa.Linn.)

Thippilimoolam (Piper longum.Linn.)

Koraikizhangu (Cyperus rotundus.Linn.)

Sadamanjil(Nardostachysgrandiflora.Linn.)

Karkadagasingi (Rhus succedanea.Linn.)

Mandooram (Ferroso ferric oxide)

Ayapodi (Ferrum)

Karisaalai juice - (Eclipta prostrate.Linn.) - Q.S

\section{PREPARATION:}

Equal quantities of the above ingredients are mixed together and the Mandooram powder is then added equal to the weight of all the chooranams. Half part of the Aya powder is then added and the ingredients are triturated with the juice of trailing eclipta (Eclipta prostrata). Pills are made into the size of a jujube (Zizyphus jujube) seed.

DOSAGE:

Jujube sized seed

\section{ADJUVANT:}

Sugar or honey

\section{INDICATIONS:}

Paandu (Anaemia)

Sobai (Anasarca)

Kamaalai (Jaundice)

Suram (Fever)

Kshayam (Tuberculosis)

\section{RESULT}

Table 1: Scientific \& selected vernacular names, families of the medicinal ingredients

\begin{tabular}{|l|l|l|l|l|}
\hline Botanical name & Tamil name & English name & Sanskrit Name & Family \\
\hline Zingiber officinalis & Chukku & Dried ginger & Nagaram & Zingiberaceae \\
\hline Piper nigrum & Milagu & Pepper & Maricha & Piperaceae \\
\hline Piper longum & Thippili & Long pepper & Pippali & Piperaceae \\
\hline Terminalia chebula & Kadukkai & Chebulic myrobalan & Pathya & Combretaceae \\
\hline Phyllanthus emblica & Nellivattral & Indian gooseberry & Amalaki & Euphorbiaceae \\
\hline Terminalia bellerica & Thandrikai & Belleric myrobalan & Vebeethaki & Combretaceae \\
\hline Elettaria cardamomum & Elam & Cardamom seeds & Ela & Zingiberaceae \\
\hline Myristica fragrans & Saadhikai & Nut meg & Jatphalam & Myrtaceae \\
\hline Syzygium aromaticum & Krambu & Clove & Lavangam & Myrtaceae \\
\hline Bauhinia tomentosa & Kattathipoo & Holy mountain ebony & Phalgu & Fabaceae \\
\hline Piper longum & Thippilimoolam & Long pepper root & Pipaleemoola & Piperaceae \\
\hline Cyperus rotundus & Koraikizhangu & Nut grass & Mutha & Cyperaceae \\
\hline Narsostachys grandiflora & Sadamanjil & Valerina root & Jatmamsi & Valerianaceae \\
\hline Rhus succedanea & Karkadagasingi & The galls & Karkatashring & Anacardiaceae \\
\hline Eclipta prostrata & Karisaalai & Trailling eclipta & Bhringaraj, Kesaranja & Asteraceae \\
\hline
\end{tabular}

Table 2: Morphology, parts used \& organoleptic charecters of the herbal ingredients

\begin{tabular}{|c|c|c|c|c|c|}
\hline Botanical name & Morphology & Parts used & Taste & Potency & Bio availability \\
\hline Zingiber officinalis & Herb & Tuber & Pungent & Hot & Pungent \\
\hline Piper nigrum & Climber & Seed & Bitter, pungent & Hot & Pungent \\
\hline Piper longum & Climber & Fruit & Sweet & Hot & Sweet \\
\hline Phyllanthus emblica & Tree & Fruit & Sour, astringent, sweet. & Cool & Sweet \\
\hline Terminalia bellerica & Tree & Fruit & Astringent & Hot & Sweet \\
\hline Syzygium aromaticum & Tree & Flower buds & Pungent & Hot & Pungent \\
\hline Bauhinia tomentosa & Tree & Flower & Bitter, astringent & Hot & Pungent \\
\hline Piper longum & Climber & Dried root & Pungent & Hot & Pungent \\
\hline Cyperus rotundus & Herb & Tuber & Slightly bitter, sweet & Cool & Pungent \\
\hline Nardostachys grandiflora & Herb & Root & Fresh piece- sweet, Dry piece- pungent & Hot & Pungent \\
\hline Rhus succedanea & Tree & Galls & Astringent & Hot & Pungent \\
\hline
\end{tabular}


Table 3: Information about metal \& mineral drugs of ThirikadugadhiMandooram

\begin{tabular}{|l|l|l|l|l|l|}
\hline Tamil name & English name & Chemical name & Taste & Potency & Biotrans formations \\
\hline Mandooram & $\begin{array}{l}\text { Iron rust, impure oxide of } \\
\text { Iron, Magnitite }\end{array}$ & $\begin{array}{l}\text { Ferroso ferric } \\
\text { oxide }\end{array}$ & Astringent & Hot & Pungent \\
\hline Ayam & Iron & Ferrum & Astringent, mild sour, bitter & Hot & Pungent \\
\hline
\end{tabular}

Table 4: Information about ingredients of Thirikadugadhi Mandooram

\begin{tabular}{|c|c|c|c|}
\hline Ingredients & Actions & Phyto chemistry & Medicinal uses in siddha \\
\hline $\begin{array}{l}\text { Zingiber } \\
\text { officinalis }\end{array}$ & Stomachic, carminative, stimulant & $\begin{array}{ll}\begin{array}{l}\text { Gingerdione, } \\
\text { gingerols, zingiberin }\end{array} & \text { shogaols, }\end{array}$ & $\begin{array}{l}\text { Indigestion, asthma, cough, anal diseases, } \\
\text { diarrhoea, anaemia, ulcer }\end{array}$ \\
\hline Piper nigrum & $\begin{array}{l}\text { Acrid, carminative, anti- vadha, } \\
\text { resolvent, rubefacient }\end{array}$ & $\begin{array}{l}\text { Piperine, chavicine,piperidine, } \\
\text { limonene, pipercide, pipericide }\end{array}$ & $\begin{array}{l}\text { Anaemia, diarrhoea, ulcer, cough, hemiplegia, } \\
\text { piles, indigestion, jaundice }\end{array}$ \\
\hline Piper longum & Stimulant, carminative & $\begin{array}{l}\text { Piperine, pipernonaline, } \\
\text { piplasterol, sesamine }\end{array}$ & $\begin{array}{l}\text { Cough, asthma, tuberculosis, anaemia, head } \\
\text { ache, kabha diseases, ulcer }\end{array}$ \\
\hline $\begin{array}{l}\text { Terminalia } \\
\text { chebula }\end{array}$ & $\begin{array}{l}\text { Laxative, tonic, } \\
\text { purgative, alterative }\end{array}$ & $\begin{array}{l}\text { Chebulinic acid, chebulagic } \\
\text { acid, chebulic acid, gallic acid, } \\
\text { fatty acid, tannin }\end{array}$ & $\begin{array}{l}\text { Eye diseases, cough, dysgeusia, liver diseases, } \\
\text { vomit, ascites, fistula, TB, anaemia, ulcer, } \\
\text { leprosy, jaundice }\end{array}$ \\
\hline $\begin{array}{l}\text { Phyllanthus } \\
\text { emblica }\end{array}$ & $\begin{array}{l}\text { Astringent, refrigerant, laxative, } \\
\text { diuretic }\end{array}$ & $\begin{array}{l}\text { Ascorbic acid, gallic acid, } \\
\text { ellagic acid, tannins }\end{array}$ & $\begin{array}{l}\text { Kabha diseases, sinusitis, gonorrhoea, vomit, } \\
\text { psychiatric disorders }\end{array}$ \\
\hline $\begin{array}{l}\text { Terminalia } \\
\text { bellerica }\end{array}$ & $\begin{array}{l}\text { Astringent, expectorant, laxative, } \\
\text { tonic }\end{array}$ & $\begin{array}{l}\text { Gallic acid, ellagic acid, ethyl } \\
\text { gallate, chebullagic acid, belleric } \\
\text { acid, bellaricanin }\end{array}$ & $\begin{array}{l}\text { Asthma, hypertension, gonorrhoea, pox } \\
\text { diseases, tooth ache, cough, spider poison }\end{array}$ \\
\hline $\begin{array}{l}\text { Elettaria } \\
\text { cardamomum }\end{array}$ & Stimulant, carminative, stomachic & $\begin{array}{l}\text { Limonene, linalool, cineole, } \\
\text { campesterol, } \quad \text { methylacetate, } \\
\text { geranyl acetate }\end{array}$ & $\begin{array}{l}\text { Throat infection, cough, diarrhoea, spider } \\
\text { poison, stomach pain, sinusitis }\end{array}$ \\
\hline $\begin{array}{l}\text { Myristica } \\
\text { fragrans }\end{array}$ & $\begin{array}{l}\text { Stimulant, carminative, narcotic, } \\
\text { aromatic, aphrodisiac, tonic }\end{array}$ & $\begin{array}{l}\text { Myricetin, elemicin, essential } \\
\text { oils and fixed oil, methyleugenol }\end{array}$ & $\begin{array}{l}\text { Diarrhoea, headache, asthma, cough, stomach } \\
\text { pain, tooth ache }\end{array}$ \\
\hline $\begin{array}{l}\text { Syzygium } \\
\text { aromaticum }\end{array}$ & $\begin{array}{l}\text { Antispasmodic, } \quad \text { carminative, } \\
\text { stomachic }\end{array}$ & $\begin{array}{l}\text { Caryophyllene oxide, eugenol, } \\
\text { acetophenone, eugenol acetate, } \\
\text { methyl palmitate }\end{array}$ & $\begin{array}{l}\text { Throat infection, headache, vomit, diarrhoea, } \\
\text { ear diseases, cataract }\end{array}$ \\
\hline $\begin{array}{l}\text { Bauhinia } \\
\text { tomentosa }\end{array}$ & Anti- periodic, tonic & $\begin{array}{l}\text { Flavone, flavono glycoside, } \\
\text { flavonoids, triterpene, saponin }\end{array}$ & $\begin{array}{l}\text { Diarrhoea, cough, gonorrhoea, hypertension, } \\
\text { Sexually transmitted diseases }\end{array}$ \\
\hline Piper longum & Stomachic & $\begin{array}{l}\text { Piperine, piplastine, sesamin, } \\
\text { piperlatine, futoamide }\end{array}$ & $\begin{array}{l}\text { Cough, STD, fever,diarrhoea, sore throat, } \\
\text { vadha diseases, cardic arrest }\end{array}$ \\
\hline $\begin{array}{l}\text { Cyperus } \\
\text { rotundus }\end{array}$ & $\begin{array}{l}\text { Demulcent, vermifuge, astringent, } \\
\text { stimulant, tonic, diuretic, } \\
\text { diaphoretic, emmenagogue }\end{array}$ & $\begin{array}{l}\text { Steroids, } \begin{array}{l}\text { Phytosteroids, } \\
\text { quinones, coumarine, cardiac } \\
\text { glycosides }\end{array} \\
\end{array}$ & $\begin{array}{l}\text { Diarrhoea, fever, hypertension, vomit, scorpion } \\
\text { bite, TB, cough }\end{array}$ \\
\hline $\begin{array}{l}\text { Nardostachys } \\
\text { grandiflora }\end{array}$ & $\begin{array}{l}\text { Stimulant,anti- spasmodic, diuretic, } \\
\text { expectorant }\end{array}$ & $\begin{array}{l}\text { Jatamansin, angelicin, elemol, } \\
\text { nardol, virolin, jatamansone }\end{array}$ & $\begin{array}{l}\text { Spider poison, fever, diarrhoea, hypertension, } \\
\text { cough, asthma, eclampsia }\end{array}$ \\
\hline $\begin{array}{l}\text { Rhus } \\
\text { succedanea }\end{array}$ & $\begin{array}{l}\text { Astringent, tonic } \\
\text { digestive, expectorant, } \\
\text { cholagogue }\end{array}$ & $\begin{array}{l}\text { Tannin, palmitic acid, stearic } \\
\text { acid, arachidic acid }\end{array}$ & Diarrhoea, cough, leucoderma \\
\hline $\begin{array}{l}\text { Eclipta } \\
\text { prostrata }\end{array}$ & $\begin{array}{l}\text { Cholagogue, tonic, alterative, } \\
\text { emetic, purgative, hepato-tonic, } \\
\text { deobstruent }\end{array}$ & $\begin{array}{l}\text { Ecliphine, nicotine, tigmasterol, } \\
\text { alkaloids, pentodecanic acid, } \\
14 \text { - heptacosanal }\end{array}$ & $\begin{array}{l}\text { Anaemia, jaundice, leprosy, oedema, tooth } \\
\text { disease, cough, liver and spleen disorders, } \\
\text { filariasis }\end{array}$ \\
\hline $\begin{array}{l}\text { Ferroso } \\
\text { ferric oxide }\end{array}$ & Stomachic, tonic, alterative & - & $\begin{array}{l}\text { Fever associated with bone disorders, severe } \\
\text { anasarca\& paleness, wheezing, dropsy due to } \\
\text { abdomen enlargement, pallor, asthma, } \\
\text { gonorrhea, deranged kapha }\end{array}$ \\
\hline Ferrum & $\begin{array}{l}\text { Tonic, haemopoietic, appetite, } \\
\text { stimulant }\end{array}$ & - & $\begin{array}{l}\text { Anaemia, jaundice, leucoderma, obesity, } \\
\text { dropsy, anorexia, peptic ulcer, spermatorrhea, } \\
\text { diarrhoea and dyspepsia. }\end{array}$ \\
\hline
\end{tabular}

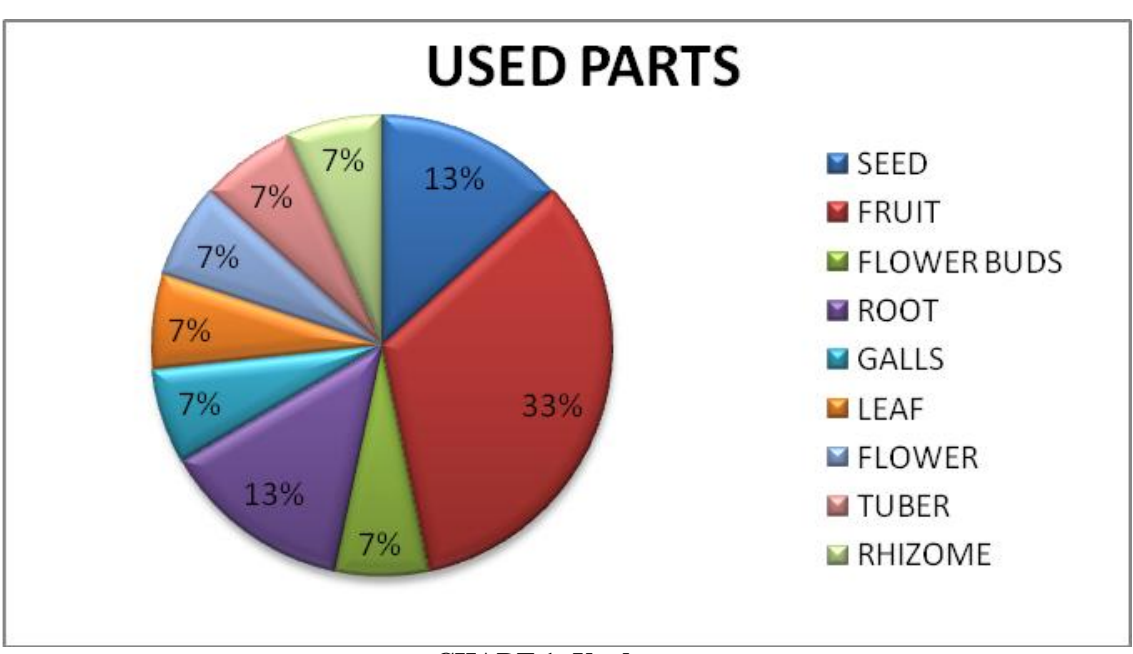

CHART 1: Used parts 


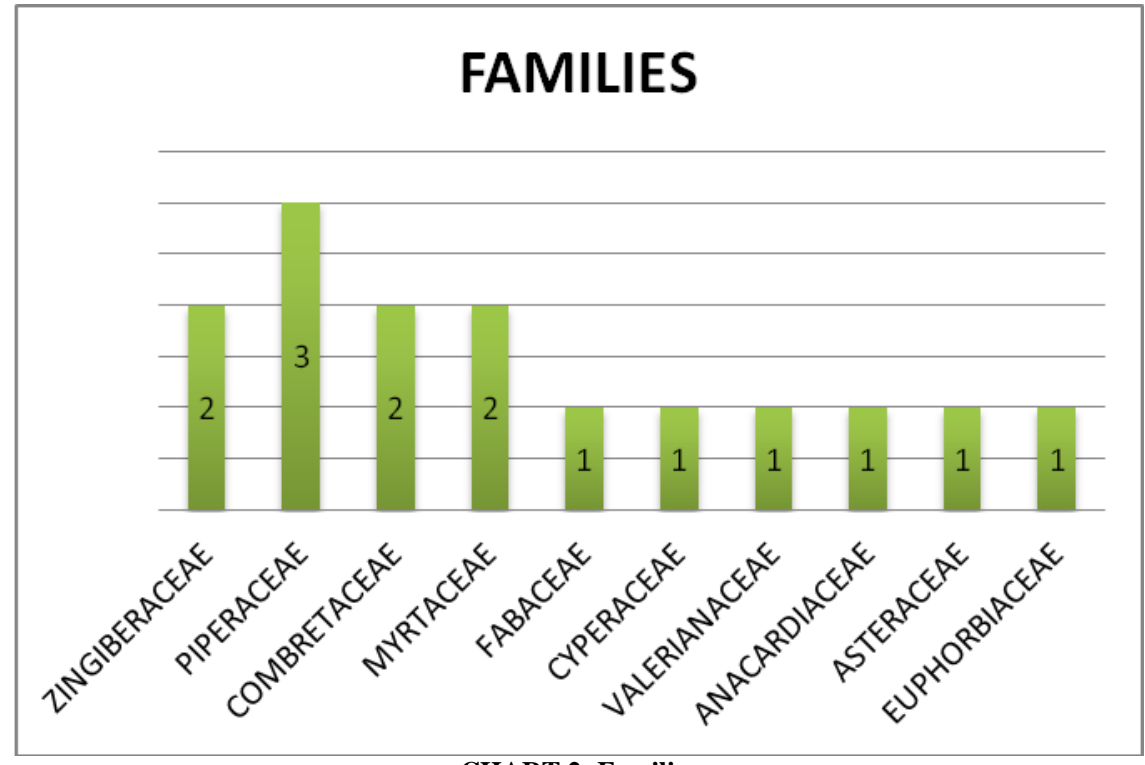

CHART 2: Families

\section{MORPHOLOGICAL CLASSIFICATION}

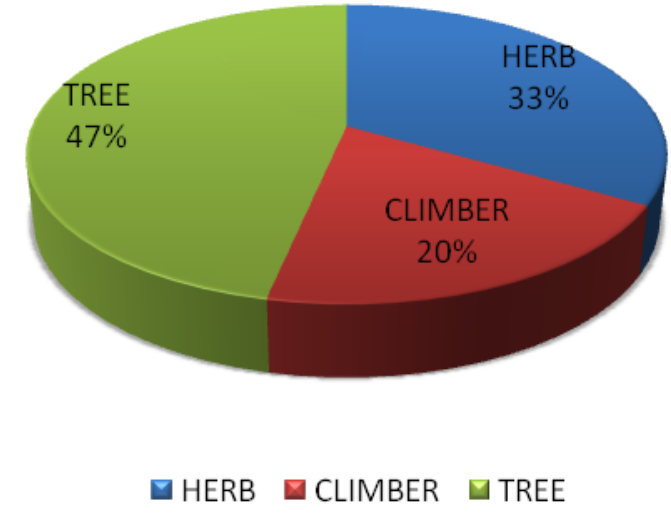

CHART 3: Morphological classification

\section{PHARMACOLOGICAL ACTION}

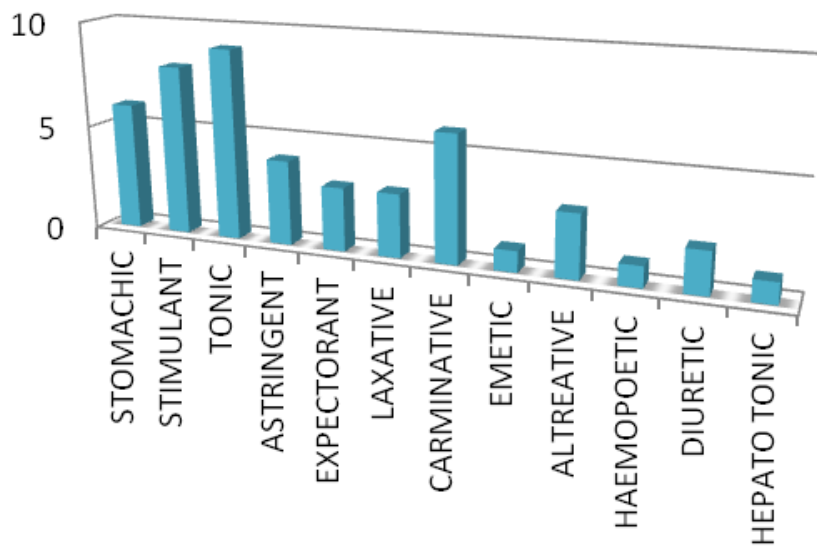




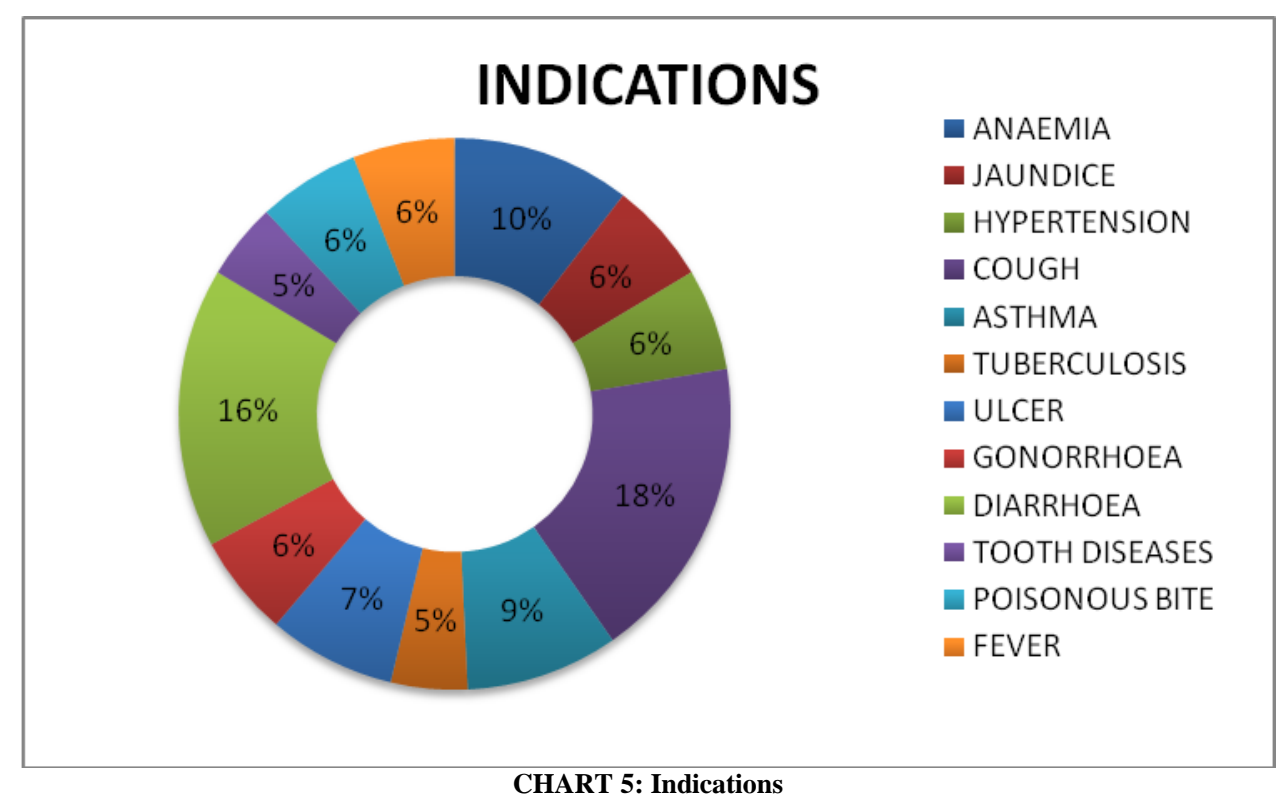

\section{DISCUSSION AND CONCLUSION}

According to the review of literature of Thirikadugadhi Mandooram, the following topics were analysed such as parts used, family, morphology, pharmacological actions and indications of each ingredients.

In parts used - fruits were used in $33 \%$, seeds \& roots were used in each $13 \%$, flower buds, flower, rhizome, tuber, leaf, galls were used in each $7 \%$. In family, 3 ingredients coming under the Piperaceae family, Zingiberaceae (2), Combretaceae (2), Myrtaceae (2), Fabaceae (1), Cyperaceae (1), Anacardiaceae (1), Asteraceae (1), Euphorbiaceae (1) were used. In the whole ingredients $47 \%$ were tree, $33 \%$ of herbs, $20 \%$ of climbers. When consider the pharmacological actions - tonic (9), stimulant (8), stomachic (6), carminative (6), astringent (4), expectorant (3), laxative (3), alterative (3), diuretic (2), haemopoietic (1), hepato tonic (1), emetic (1). Finally based on the indications, the following results were find out from the text $\&$ researches- cough $18 \%$, diarrhoea $17 \%$, anaemia $11 \%$, asthma $9 \%$, ulcer $8 \%$, jaundice $6 \%$, hypertension $6 \%$, poisonous bite $6 \%$, fever $6 \%$, gonorrhea $6 \%$, tooth diseases $5 \%$, tuberculosis $4 \%$.

In conclusion,

Based on the review, the pharmacological actions, organoleptic characters and chemical constituents of each ingredients were perfectly matched with the indications of 'Thirikadugadhi Mandooram' which mentioned in siddha text.

Acknowledgement: None

\section{Conflict of Interest: None}

\section{Source of Funding: None}

\section{REFERENCES}

1. Thiyagarajan.R, 2008, Siddha Materia Medica (Mineral \& Animal section) in Tamil, $1^{\text {st }}$ edition, Department of Indian Medicine \& Homeopathy, Chennai-106.

2. Thiru K.S.Murugesa Mudhaliyar, 2013, Gunapadam Mooligai vaguppu (part 1), Department of Indian Medicine \& Homeopathy, Chennai-106.

3. Anonymous, 2007, WHO country cooperation strategy 2006-2011 supplement on traditional medicine. New Delhi.

4. Sambasivampillai .T.V., 2016, Siddha Medical Dictionary (Tamil-English), $4^{\text {th }}$ print, Department of Indian Medicine \& Homeopathy, Chennai-106

5. Vaidhyaratnam P S, 1989, Variers's, Indian Medicinal Plants - a compendium of 500 species - vol-5, Arya vaidhya sala, Kottakal

6. Somasundaram.S, 2015, Taxonomy Of Angiosperms (Medicinal plants part-2), $6^{\text {th }}$ edition, Ilangovan publishers, Tirunelveli627002

7. Anonymous, 2011, The Siddha Pharmacoepia of India, part 1, vol-1\&2, 
Depart of Indian Medicine \& Homeopathy, New Delhi,.

8. Anaivaari Ananthanan, 2008, Sarakku Suddhi Sei Muraigal, $1^{\text {st }}$ edition, Department of Indian Medicine \& Homeopathy, Chennai

9. Ambai Arunachalam, Mooligaiiyal, Gandhimathi Printers, Tirunelveli-627 003.

10. Kumarasamy.R, 1978, Medical Taxonomy (part 1\&2), Indian Medical College, Palyamkottai

11. Kandaswamy pillai.N, 2012, History of Siddha Medicine, $3^{\text {rd }}$ edition, Department of Indian Medicine \& Homeopathy, Chennai.
12. Gupta A.K., 1988, Wealth of India, vol-4, Publications \& Information Directorate, New Delhi.

13. Pulliah.T, 2002, Medicinal plants in India vol-1, Regency publications, New Delhi.

How to cite this article: Shakthi Priya M, Essakky Pandian G. Drug review on siddha drug - thirikadugadhi mandooram. Int $J$ Health Sci Res. 2021; 11(5): 140-145. DOI: https://doi.org/10.52403/ijhsr.20210521 